\title{
Interaction between perineuronal nets and ketamine in antidepressant action
}

\author{
Calvin K. Young, Kachina G. Kinley and Neil McNaughton
}

Dept. Psychology and Brain Health Research Centre, University of Otago, Dunedin, New Zealand

ORCID IDs: $\mathrm{CKY}=0000-0002-6130-8370 ; \mathrm{NMcN}=0000-0003-4348-8221$

Running head: PNN and antidepressant action

Correspondence:

Calvin K. Young

Department of Psychology,

University of Otago

PO BOX 56, Dunedin, New Zealand

Telephone: +64-3-479-7621

Fax: $\quad+64-3-479-8335$

Email: $\quad$ calvin.young@otago.ac.nz 


\begin{abstract}
Depression is highly prevalent, increases suicide risk, and is now the leading cause of disability worldwide. Our ability to treat depression is hampered by the lack of understanding of its biological underpinnings and of the mode of action of effective treatments. We hypothesised that the scaffolding proteins in the medial frontal cortex play a major role in effective antidepressant action. We implanted cannulae into the infralimbic cortex to inject chABC and locally remove perineuronal nets and then tested for antidepressant effects with the forced swim test. We further tested if systemic injections of ketamine had an additive effect. Our preliminary data indicate that neither the removal of these scaffolding proteins nor ketamine were sufficient to decrease depression-like behaviour, but may interact synergistically to decrease immobility time in the forced swim test.
\end{abstract}

Keywords: perineuronal nets; depression; antidepressant; ketamine; prefrontal cortex; rat; behaviour 


\section{Introduction}

Depression is on the rise worldwide (WHO 2017). This trend warrants attention as depression can significantly decrease quality of life, is associated with poorer prognosis of other conditions, and places heavy demands on the healthcare system (Roy-Byrne, Stang et al. 2000, Oakley-Browne, Wells et al. 2006, Brakowski, Spinelli et al. 2017). There are various treatments for depression, including antidepressant medication and cognitive behavioural therapy. However, we know little about the biological basis of the disorder and about how effective treatments work.

Current first-line treatment is through modulation of the monoamine system. The effectiveness of monoamine treatments, particularly commonly used selective serotonin reuptake inhibitors (SSRIs) such as Prozac, suggests that patients with depression have reduced levels of serotonin, dopamine, and/or norepinephrine (Hirschfeld 2000). However, alternative forms of treatment including prefrontal cortex deep brain stimulation, electric shock therapy, and cognitive behaviour therapy have proven to be effective, even though they do not appear to act directly on the monoamine system. One thing all these treatments have in common is that they increase synaptic plasticity by increasing brain-derived neurotrophic factor - BDNF (Nibuya, Morinobu et al. 1995, Gersner, Toth et al. 2010, Duman and Aghajanian 2012). Thus, the aetiology of depression and its effective treatment may hinge on quantifiable wiring abnormalities in the brain.

A growing literature implicates perineuronal nets (PNN) in psychiatric conditions (Berretta 2012). PNNs are organised structures composed of chondroitin sulfate proteoglycans (CSPG) and extracellular matrix (Deepa, Carulli et al. 2006), which is crucial in stabilising neural connections (Bahia, Houzel et al. 2008). Degradation of GSPGs by the enzyme chondroitinase $A B C$ (chABC) has been 
shown to assist in recovery from spinal cord injury (Bradbury, Moon et al. 2002), promote the erasure of traumatic memories (Gogolla, Caroni et al. 2009), and reverse developmental critical periods (Pizzorusso, Medini et al. 2002).

SSRIs (the most commonly prescribed antidepressant medication) have demonstrated effects on PNNs (Maya Vetencourt, Sale et al. 2008, Karpova, Pickenhagen et al. 2011, Scali, Begenisic et al. 2013); producing reduced PNN staining in the mPFC, which suggests that PNNs are altered by the antidepressant effects of the treatment (Ohira, Takeuchi et al. 2013).

Ketamine has long-lasting antidepressant effects (Browne and Lucki 2013) that, unlike SSRIs, are immediate. Ketamine is also effective in those resistant to other antidepressant treatments (Gill, Gill et al. 2021). However, it has not been tested whether or not ketamine modifies PNNs in the mPFC relating to depressionlike behaviours. Here we tested whether the degradation of PNNs underlies antidepressant treatment by allowing greater freedom for synaptic re-wiring. We probe how systemic ketamine changes both PNNs and depression-like behaviours in rodents. Additionally, we examine whether the direct, non-pharmacological removal of PNNs in the mPFC using chABC results in antidepressant behavioural effects.

\section{Methods}

Sixteen male Wistar rats $(350-430 \mathrm{~g})$ obtained from the Department of Laboratory Animal Sciences at the University of Otago received bilateral guide cannula implantation targeting the infralimbic cortex (IL; AP: +3.2 mm; ML: +/- $1.4 \mathrm{~mm} @ 10^{\circ}$; DV: $-4.7 \mathrm{~mm}$ ) after seven days of acclimatisation. After a seven-day recovery period, each rat was subjected to a 15 -minute swim session as part of the forced swim test (Slattery and Cryan 2012). Thirty minutes after the swim, rats were first injected with 
$0.5 \mu$ control enzyme (penicillinase, P0389-1KU, Sigma, USA) or chABC (C366710UN, Sigma, USA) through an injection cannula inserted to the guides (i.e. into the IL) at a rate of $\sim 0.3 \mu \mathrm{l} / \mathrm{min}$. The injection cannulae were left in place for 5 minutes after the injection. Injections were always made first in the right hemisphere, then the left. Immediately after this, $10 \mathrm{mg} / \mathrm{kg}$ of ketamine (ket) or volume-matched physiological saline (sal; 0.9\%) was injected subcutaneously. This yielded a $2 \times 2$ design with 4 rats in each group receiving a combination of ketamine/saline (ket/sal) and penicillinase/chABC (pen/chABC) injections. The rats were monitored for at least 30 minutes after the last injection. Twenty-three hours later, rats were exposed to another five-minute swim session. Thirty minutes after the second exposure, rats were transcardially perfused with phosphate-buffered saline followed by $4 \%$ paraformaldehyde (PFA, in $0.1 \mathrm{M}$ phosphate buffer). The extracted brains were further fixed in 4\% PFA for 24 hours, transferred to a $30 \%$ sucrose (in phosphatebuffered saline) solution for cryoprotection prior to sectioning. Forty-micron sections were made in a cryostat and stored in Tris-buffered saline (TBS) prior to immunostaining.

Immunostaining of PNNs and parvalbumin positive interneurons $(\mathrm{PV}+)$ were visualised by WFA (Wisteria floribunda agglutinin) conjugated fluorescein (VEFL1351, Vector Laboratories, USA) and rabbit anti-parvalbumin (PV24, Swant, Switzerland) antibody. Sections were first washed with TBS, followed by incubation in a blocking solution ( $3 \%$ normal goat serum; NGS, and $0.03 \%$ Triton-X) to reduce background staining and increase the permeability of the tissue. WFA-fluorescein (1:1000) and rabbit anti-PV (1:1000) were added to TBS+3\% NGS to incubate overnight. Additional washes in TBS were made before incubation with a goat antirabbit Cy3 secondary antibody (1:200; 111-165-144 1:200, Jackson 
ImmunoResearch, USA) for 4 hours. Final TBS washes were made before sections were mounted on pre-cleaned glass slides and coverslipped with anti-fade glue (F6057, Sigma, USA) with DAPI (a nuclear stain).

Microscope images were acquired with a Nikon (C2plus) confocal microscope at 20x magnification. Two laser lines (488 $\mathrm{nm}$ and $561 \mathrm{~nm}$ ) were used to excite WFA-fluoroscein and PV (Cy3), respectively. We centred our field of view aimed at the prelimbic cortex (PrL, immediately dorsal to IL) and collected a montage (between $7 \times 9$ to $9 \times 13$ grids, at $512 \times 512$ pixels per grid) with a stack of 9 images in 1 micron steps in the z-axis. The acquired images were imported to ImageJ/FIJI (https://fiji.sc, NIH, USA) for further analysis. Firstly, the pseudo-coloured images were pre-processed into 8-bit greyscale images. Regions of interest (ROI) were drawn around IL where this was free of damage caused by the cannulae. Four ROls (two from each hemisphere) were exported in this fashion for each rat. These images were submitted to semi-automated analysis using PIPSQUEAK (Slaker, Harkness et al. 2016). Thresholds of detection were manually adjusted to include most, if not all of the signal in each channel (i.e. WFA and PV). Auto-fluorescence was manually removed, and missed positive staining was added before single-cell ROls were processed for single/double labelling analysis.

Statistical analyses were carried out in SPSS (IBM, USA). Data transformation, curve fitting, and figure preparation were performed in Matlab (MathWorks, USA). For the FST, the number of right hind leg kicks per second was taken as the quantitative measure for swimming activity (Warden, Selimbeyoglu et al. 2012). Particularly, a distinction was made between full kicks and half kicks, with the latter more prominent in attempts to stay afloat. This measure was averaged in 5 second periods to reduce variability, and any bin returning less or equal to 1 was 
counted as immobile. In addition, immobility latency was calculated as the midpoint of a sigmoidal fit of the "kick-frequency" data over time. The swim-to-float curves of four rats' (2 from the ket/pen group, and 2 from the sal/pen group) did not conform with a sigmoidal curve, and the midpoint of a logarithmic function was used in these cases instead. Histological results were exported as a text file, which included the number of cells detected and background-subtracted intensity used here for further analysis. For both datasets, two-way analyses of variance (ANOVA) were carried out. Pearson correlation coefficients are reported for the relationship between behaviour and histological findings. One rat (ket/chABC group) died due to surgical complications and another rat (sal/pen group) had unusable brain sections due to high background fluorescence; the latter rat contributed to FST data but not histological data.

\section{Results}

First, we demonstrated that the cannula tips were located within the IL region (e.g. Figure 1A). Cannula tips from only one rat from the ket+pen group could not be located (damage to the frontal cortex by dura during brain extraction) but were presumed to be in the PrL/IL region from available histology. Figure $1 \mathrm{~A}$ also shows strong colocalization (yellow) between WFA (green) and PV (red); in contrast to chABC-digested tissue (Figure 1B) where darker patches, as well as a red (PV-only) signal can be seen around the injected area and along the guide cannula tracks. At 60x magnification (Figure 1C), colocalization was clear with most of the PV neurons WFA+. A zoomed-in example of chABC-treated tissue (Figure 1D) is shown with a white line placed along the border of the PNN digest. The left side is more ventral to the cannula tip, and WFA staining appears to be unaltered. In contrast, the right side 
is immediately below the cannula track and clearly lacks WFA (green) staining in the presence of PV (red).

The amount of immobility in the FST was highly variable. Although we did observe the lowest variance and mean value in the ket/chABC condition (Figure 2A), there was no interaction between systemic (i.e. sal/ket) and intracerebral (pen/chABC) administration $\left(F_{(1,10)}=2.299, p=.16\right)$, or any effect of chABC or ketamine on their own $\left(F_{(1,10)}=.997, p=.34\right.$ and $F_{(1,10)}=1.150, p=.31$, respectively). Although variance again was low for the ket/chABC group (Figure 2B), there is no effect of ketamine, chABC, or a combination of the treatments $\left(F_{(1,10)}=\right.$ $.008, p=.93, \mathrm{~F}_{(1,10)}=1.354, p=.27, \mathrm{~F}_{(1,10)}=.623, p=.45$, respectively) .

As expected, none of our treatments altered the expression of PV, with the number of $\mathrm{PV}+$ neurons (Figure $3 \mathrm{~A}$ ), total area of $\mathrm{PV}$ signal (Figure $3 \mathrm{~B}$ ) and background-corrected intensity (Figure 3C) all comparable (all tests are nonsignificant, $p>.13)$. Digestion of PNNs with chABC did decrease the association of PV+ neurons with WFA (Figure 3D; $F_{(1,10)}=16.017, p<.005$ ) with no effect of ketamine $\left(F_{(1,10)}=.33, p=.86\right)$ or the combination of chABC with ketamine $\left(F_{(1,10)}=\right.$ $.002, p=962)$.

In contrast, chABC greatly reduced the density of WFA staining (Figure 4A; $\left.F_{(1,10)}=22.021, p<.001\right)$ with no interaction with ketamine injection $\left(F_{(1,10)}=.59, p=\right.$ .46). There is no effect of ketamine on the density of WFA staining $\left(F_{(1,10)}=391, p=\right.$ .55). The average area of counted WFA staining was not different for chABC, ketamine or a combination of treatments (Figure 4B; all tests non-significant, $p>$ .29). Mirroring changes seen in WFA density, the background-corrected intensity of the WFA signal was decreased after chABC treatment (Figure4C; $F_{(1,10)}=12,711, p$ $<.005)$ but not after ketamine $\left(F_{(1,10)}=1.307, p=.28\right)$, with no interaction between 
the two treatments $\left.F_{(1,10)}=.253, p=.63\right)$. There was no difference in the ratio of WFA+ neurons that were also PV+ (Figure 4D; all tests non-significant, $p>.27)$.

Although there was a clear histological outcome for chABC treatment, the behavioural outcome was inconclusive. To account for potential differences in the extent of chABC digest in relation to behaviour, we carried out correlation analysis in an attempt to establish possible dependencies. We found no relationship between the time spent immobile in the FST and: 1) the density of WFA staining (Pearson's $r(14)=-.04, p=.89) ; 2)$ the proportion of $\mathrm{PV}+$ that are also WFA+ (Pearson's $r(14)=$ $.01, p=.98)$ or; 3 ) the proportion of WFA+ neurons that are PV+ (Pearson's $r(14)=$ $.16, p=.58)$. Although correlations of the same histological measurements with immobility latency was more consistent with the hypothesised relationship, there was no detected significant correlation with: 1 ) density of WFA staining (Pearson's $r(14)=$ $.38, p=.18) ; 2)$ the proportion of PV+ that are also WFA+ (Pearson's $r(14)=.32, p$ $=.26)$ or; 3$)$ the portion of WFA+ neurons that are PV+ (Pearson's $r(14)=-.01, p=$ $.98)$.

\section{Discussion}

In the current study, we sought to establish a direct link between the integrity of PNNs and depression-like behaviour in the FST. Our data indicate that $10 \mathrm{mg} / \mathrm{kg}$ ketamine was not effective as an antidepressant in naïve rats tested with FST, and did not change IL WFA staining. We did not observe any antidepressant effect of PNN removal in the $\mathrm{IL}$ and surrounding areas, despite an almost complete elimination of PNNs.

We were not able to replicate various studies showing ketamine having an antidepressant effect at sub-anaesthetic doses (i.e. $10 \mathrm{mg} / \mathrm{kg}$ ) injected 24 hours prior 
to the test (Fukumoto, Toki et al. 2017, Browne, Falcon et al. 2018, Wan, Feng et al. 2018). We were also not able to demonstrate a clear effect of chABC on depressionlike behaviour in the FST. However, we do note that ketamine+chABC treatment did seem to yield consistently favourable results in relation to our hypothesis but the effect was not found to be statistically significant. One obvious weakness of the current study is the low number of samples in each condition. Previous studies have used a minimum of $8+$ animals (Fukumoto, Toki et al. 2017, Browne, Falcon et al. 2018, Wan, Feng et al. 2018) to obtain statistically significant differences between control and experimental groups. Our study is somewhat underpowered to sufficiently answer our research questions, but additional data is currently being analysed to clarify the findings described here. We note recent studies have highlighted the relevance of stress levels in rodents relating to ketamine's antidepressant effects. Specifically, ketamine increased axiogenic behaviours and decreased social interaction time as would stress-induced depression models in naïve Wistar rats (Silvestre, Nadal et al. 1997). In mice, ketamine produced antidepressant effects in stressed mice but had the opposite effect in non-stressed mice (Fitzgerald, Yen et al. 2019, Trofimiuk, Wielgat et al. 2019), an effect also observed in humans (Nugent, Ballard et al. 2019). Despite high variance, our behavioural data indicated an overall pro-depressant effect as measured by immobility in FST. An expanded behavioural battery and the manipulation of pretreatment stress levels will shed more light on the complex interaction between stress, treatment outcome and the role of structural plasticity.

Traditionally, FST scoring involves the scorer assessing the "dominant" behaviour in $5 \mathrm{~s}$ bins to provide a single binary assessment (i.e. immobility or otherwise) of animals' activity in the 5 min test (Slattery and Cryan 2012). As an 
attempt to move away from subjective measures of FST behaviour, we employed a more objective measure of hind leg kicks in FST (Warden, Selimbeyoglu et al. 2012). However, we note that our division of full kicks (full extension of the hind leg) and half kicks (less than full extension of the hind leg) does involve a certain degree of subjectivity. Regardless, it is important to point out that we did not score the FST in a conventional way, thus it is unclear if the lack of effect may be due to scoring differences. In addition, we did not differentiate "climbing" (vertical movement) and "swimming" (horizontal movement) behaviours in our current attempt. We are currently applying alternative algorithms that can automatically score behaviour more objectively (Yuman, Idaku et al. 2008), and/or use deep learning algorithms (Mathis, Mamidanna et al. 2018) to carry out more detailed kinematic analyses of FST behaviour.

It is unclear what effect, if any, the damage as a result of cannula implant/intracerebral injection has on PNN/PV expression and FST behaviour. We targeted the IL due to its apparent central role in depression and antidepressant action (Covington, Lobo et al. 2010, Scopinho, Scopinho et al. 2010, Slattery, Neumann et al. 2011, Seese, Chen et al. 2013, Chang, Chen et al. 2014, Fuchikami, Thomas et al. 2015). IL inactivation and lesion have produced opposite effects on FST immobility in past studies (Scopinho, Scopinho et al. 2010, Slattery, Neumann et al. 2011, Chang, Chen et al. 2014). Our short implant to testing time window (5-7 days), as well as potential additional damage done by insertion/removal of the dummy and injection cannulae 24 hours before proper FST testing has not been adequately controlled for. Additional data are needed to account for the potential acute effects of lesion/injections into the IL area on FST behaviour. In addition to potential issues lesions impose on behaviour, their effects on histological analysis 
are evident. Although we were able to obtain data from the IL by using sections that immediately surrounding the damaged tissue (where injections were made) it is clear that the damaged area is not uniform across rats and thus creates variation in where exactly viable histological analyses could be carried out. In future studies, PNN modification relating to ketamine may have to be done independently to avoid confounds caused by local lesions. However, similar issues relating to localised intracerebral PNN digest cannot be solved in the same way. The use of biocompatible hydrogels (Clarkson, Parker et al. 2015) for slow release or a combination of transgenic/viral vector techniques (Gossen and Bujard 1992, Madisen, Zwingman et al. 2010, Zhao, Muir et al. 2011) may be used to circumvent the effects of lesions relating to the use of implanted cannulae described here.

The hippocampus and the amydgala, two areas that have been found to undergo activity changes in depression (Zeng, Shen et al. 2012) may reveal further insights to the effectiveness of our manipulations without the confounds described in IL above due to tissue damage.

To summarise, we failed to replicate the therapeutic effects of ketamine on depression-like behaviour in the FST. Consistent with the lack of effect, we found no evidence of PNN modification by systemic ketamine injections. Our hypothesis of PNN removal being antidepressant is not fully supported; only a combination of systemic ketamine and IL chABC injection resulted in a trend to an antidepressant effect, despite dramatic removal of PNNs by chABC in the IL and surrounding regions. Together, our data provide preliminary clues on how ketamine and chABC may act synergistically to produce antidepressant effects. Additional experiments are currently underway to provide adequate statistical power for firmer conclusions. 


\section{Acknowledgements}

KGK was supported by a summer research scholarship by the Neurological Foundation of New Zealand. The project was supported by a University of Otago Research Grant.

\section{Contributions}

CKY conceived the study, carried out experiments, confocal image acquisition and data analyses. KGK carried out behavioural scoring, immunohistochemistry, confocal image acquisition and image analysis. NM provided supervision and secured funding for the research. CKY prepared the manuscript and all authors reviewed and edited the manuscript.
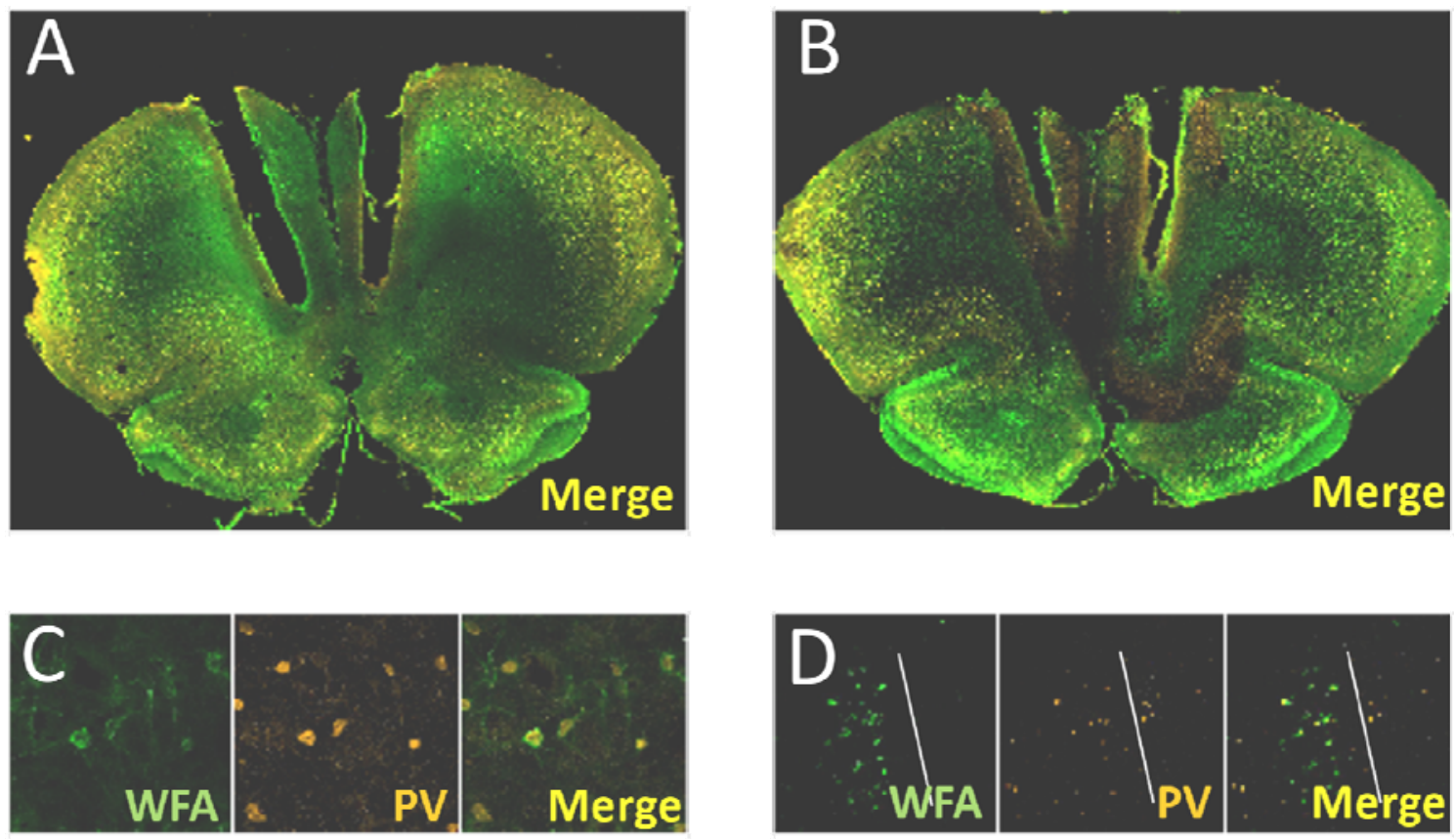

Figure 1. PNN digest with chABC with WFA and PV staining. A: An example of a vehicle-injected prefrontal area with WFA and PV staining intact compared to B: 
chABC injected tissue, where clear elimination of WFA signal around the cannula track can be seen. C: 20x magnification of representative co-staining of WFA and PV, and D: zoomed in view of PV staining without WFA at the "border" (white line) of chABC digest.

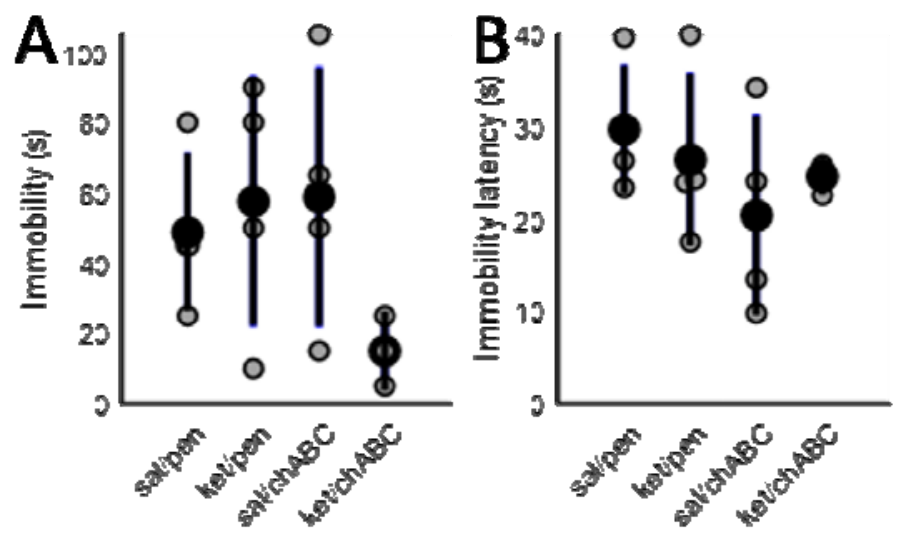

Figure 2. Immobility measures in the FST. A: Mean and SE (black) for the time spent immobile in the FST and; B: latency to first bout of immobility.

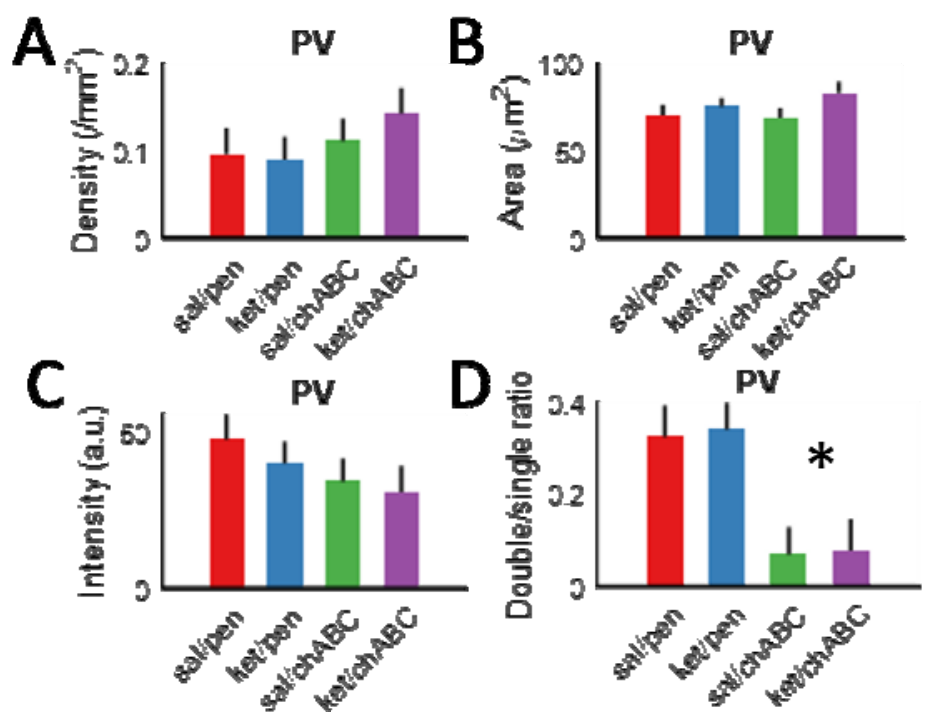

Figure 3. Characteristics of PV staining in the IL. A: PV cell count density, B: total area (sum of cell sizes), C: fluorescence intensity, and D: ratio of PV (single) and PV+WFA (double) stained cell counts. 

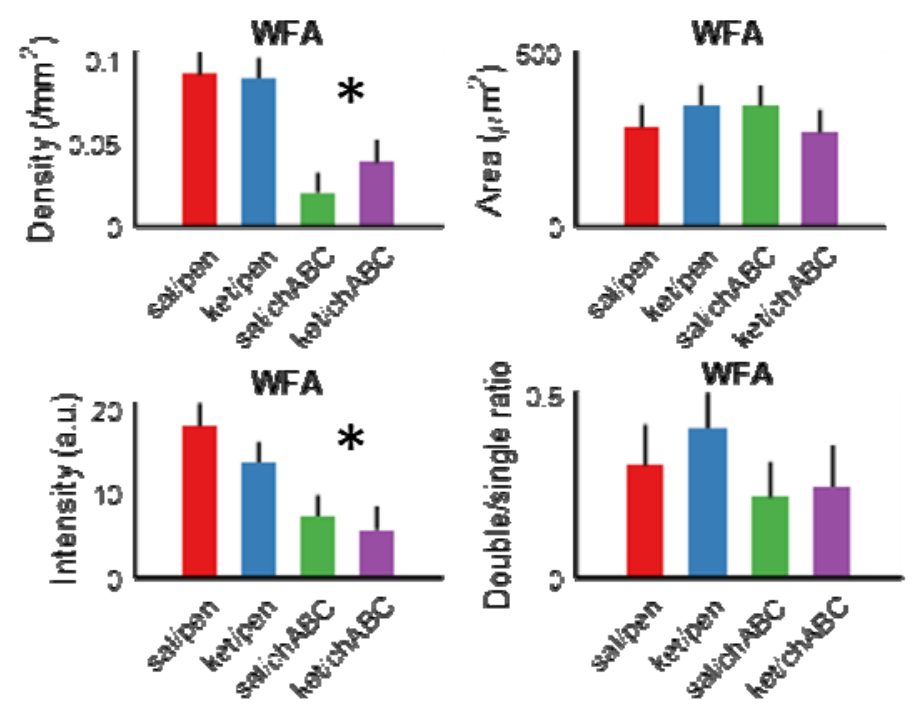

Figure 4. Characteristics of WFA staining in the IL. A: WFA cell count density, B: total area (sum of cell sizes), C: fluorescence intensity, and D: ratio of WFA (single) and WFA+PV (double) stained cell counts.
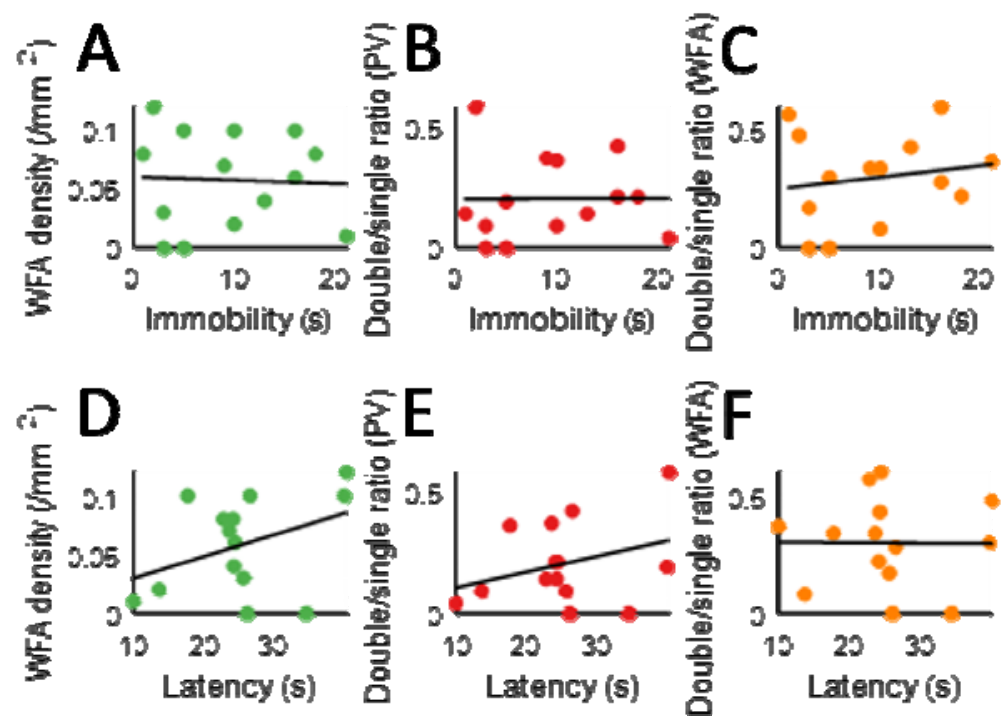

Figure 5. Relationship between WFA/PV staining and immobility in the FST.

Correlaitons between A: WFA density, B: PV+WFA/PV labelling ratio, and $\mathbf{C}$ : PV+WFA/PV labelling ratio and time spent immobile in the FST. D-F: Same histological measures correlated against the latency to first bout of immobility in the 
FST.

\section{References}

Bahia, C. P., J. C. Houzel, C. W. Picanco-Diniz and A. Pereira, Jr. (2008). "Spatiotemporal distribution of proteoglycans in the developing rat's barrel field and the effects of early deafferentation." $\mathrm{J}$ Comp Neurol 510(2): 145-157.

Berretta, S. (2012). "Extracellular matrix abnormalities in schizophrenia." Neuropharmacology 62(3): 1584-1597.

Bradbury, E. J., L. D. Moon, R. J. Popat, V. R. King, G. S. Bennett, P. N. Patel, J. W. Fawcett and S. B. McMahon (2002). "Chondroitinase ABC promotes functional recovery after spinal cord injury." Nature 416(6881): 636-640.

Brakowski, J., S. Spinelli, N. Dorig, O. G. Bosch, A. Manoliu, M. G. Holtforth and E. Seifritz (2017). "Resting state brain network function in major depression - Depression symptomatology, antidepressant treatment effects, future research." J Psychiatr Res 92: 147-159.

Browne, C. A., E. Falcon, S. A. Robinson, O. Berton and I. Lucki (2018). "Reversal of Stress-Induced Social Interaction Deficits by Buprenorphine." Int J Neuropsychopharmacol 21(2): 164-174.

Browne, C. A. and I. Lucki (2013). "Antidepressant effects of ketamine: mechanisms underlying fastacting novel antidepressants." Front Pharmacol 4: 161.

Chang, C. H., M. C. Chen, M. H. Qiu and J. Lu (2014). "Ventromedial prefrontal cortex regulates depressive-like behavior and rapid eye movement sleep in the rat." Neuropharmacology 86: 125-132. Clarkson, A. N., K. Parker, M. Nilsson, F. R. Walker and E. K. Gowing (2015). "Combined ampakine and BDNF treatments enhance poststroke functional recovery in aged mice via AKT-CREB signaling." J Cereb Blood Flow Metab 35(8): 1272-1279.

Covington, H. E., 3rd, M. K. Lobo, I. Maze, V. Vialou, J. M. Hyman, S. Zaman, Q. Laplant, E. Mouzon, S. Ghose, C. A. Tamminga, R. L. Neve, K. Deisseroth and E. J. Nestler (2010). "Antidepressant effect of optogenetic stimulation of the medial prefrontal cortex." J Neurosci 30(48): 16082-16090.

Deepa, S. S., D. Carulli, C. Galtrey, K. Rhodes, J. Fukuda, T. Mikami, K. Sugahara and J. W. Fawcett (2006). "Composition of perineuronal net extracellular matrix in rat brain: a different disaccharide composition for the net-associated proteoglycans." J Biol Chem 281(26): 17789-17800.

Duman, R. S. and G. K. Aghajanian (2012). "Synaptic dysfunction in depression: potential therapeutic targets." Science 338(6103): 68-72.

Fitzgerald, P. J., J. Y. Yen and B. O. Watson (2019). "Stress-sensitive antidepressant-like effects of ketamine in the mouse forced swim test." PLoS One 14(4): e0215554.

Fuchikami, M., A. Thomas, R. Liu, E. S. Wohleb, B. B. Land, R. J. DiLeone, G. K. Aghajanian and R. S. Duman (2015). "Optogenetic stimulation of infralimbic PFC reproduces ketamine's rapid and sustained antidepressant actions." Proc Natl Acad Sci U S A 112(26): 8106-8111.

Fukumoto, K., H. Toki, M. lijima, T. Hashihayata, J. I. Yamaguchi, K. Hashimoto and S. Chaki (2017). "Antidepressant Potential of (R)-Ketamine in Rodent Models: Comparison with (S)-Ketamine." $\underline{J}$ Pharmacol Exp Ther 361(1): 9-16.

Gersner, R., E. Toth, M. Isserles and A. Zangen (2010). "Site-specific antidepressant effects of repeated subconvulsive electrical stimulation: potential role of brain-derived neurotrophic factor." Biol Psychiatry 67(2): 125-132.

Gill, H., B. Gill, N. B. Rodrigues, O. Lipsitz, J. D. Rosenblat, S. El-Halabi, F. Nasri, R. B. Mansur, Y. Lee and R. S. Mclntyre (2021). "The Effects of Ketamine on Cognition in Treatment-Resistant Depression: A Systematic Review and Priority Avenues for Future Research." Neurosci Biobehav Rev 120: 78-85.

Gogolla, N., P. Caroni, A. Luthi and C. Herry (2009). "Perineuronal nets protect fear memories from erasure." Science 325(5945): 1258-1261.

Gossen, M. and H. Bujard (1992). "Tight control of gene expression in mammalian cells by tetracycline-responsive promoters." Proc Natl Acad Sci U S A 89(12): 5547-5551.

Hirschfeld, R. M. (2000). "History and evolution of the monoamine hypothesis of depression." J Clin Psychiatry 61 Suppl 6: 4-6.

Karpova, N. N., A. Pickenhagen, J. Lindholm, E. Tiraboschi, N. Kulesskaya, A. Agustsdottir, H. Antila, D. Popova, Y. Akamine, R. Sullivan, R. Hen, L. J. Drew and E. Castren (2011). "Fear erasure in mice requires synergy between antidepressant drugs and extinction training." Science 334(6063): 17311734. 
Madisen, L., T. A. Zwingman, S. M. Sunkin, S. W. Oh, H. A. Zariwala, H. Gu, L. L. Ng, R. D. Palmiter, M. J. Hawrylycz, A. R. Jones, E. S. Lein and H. Zeng (2010). "A robust and high-throughput Cre reporting and characterization system for the whole mouse brain." Nat Neurosci 13(1): 133-140. Mathis, A., P. Mamidanna, K. M. Cury, T. Abe, V. N. Murthy, M. W. Mathis and M. Bethge (2018). "DeepLabCut: markerless pose estimation of user-defined body parts with deep learning." Nat Neurosci 21(9): 1281-1289.

Maya Vetencourt, J. F., A. Sale, A. Viegi, L. Baroncelli, R. De Pasquale, O. F. O'Leary, E. Castren and L. Maffei (2008). "The antidepressant fluoxetine restores plasticity in the adult visual cortex." Science 320(5874): 385-388.

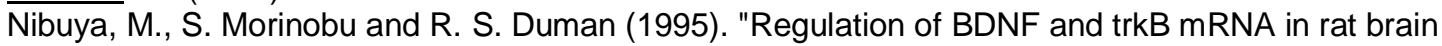
by chronic electroconvulsive seizure and antidepressant drug treatments." J Neurosci 15(11): 75397547.

Nugent, A. C., E. D. Ballard, T. D. Gould, L. T. Park, R. Moaddel, N. E. Brutsche and C. A. Zarate, Jr. (2019). "Ketamine has distinct electrophysiological and behavioral effects in depressed and healthy subjects." Mol Psychiatry 24(7): 1040-1052.

Oakley-Browne, M. A., J. E. Wells and K. M. Scott, Eds. (2006). Te Rau Hinengaro: The New Zealand Mental Health Survey. Wellington, Ministry of Health.

Ohira, K., R. Takeuchi, T. Iwanaga and T. Miyakawa (2013). "Chronic fluoxetine treatment reduces parvalbumin expression and perineuronal nets in gamma-aminobutyric acidergic interneurons of the frontal cortex in adult mice." Mol Brain 6: 43.

Pizzorusso, T., P. Medini, N. Berardi, S. Chierzi, J. W. Fawcett and L. Maffei (2002). "Reactivation of ocular dominance plasticity in the adult visual cortex." Science 298(5596): 1248-1251.

Roy-Byrne, P. P., P. Stang, H. U. Wittchen, B. Ustun, E. E. Walters and R. C. Kessler (2000).

"Lifetime panic-depression comorbidity in the National Comorbidity Survey. Association with symptoms, impairment, course and help-seeking." Br J Psychiatry 176: 229-235.

Scali, M., T. Begenisic, M. Mainardi, M. Milanese, T. Bonifacino, G. Bonanno, A. Sale and L. Maffei (2013). "Fluoxetine treatment promotes functional recovery in a rat model of cervical spinal cord injury." Sci Rep 3: 2217.

Scopinho, A. A., M. Scopinho, S. F. Lisboa, F. M. Correa, F. S. Guimaraes and S. R. Joca (2010). "Acute reversible inactivation of the ventral medial prefrontal cortex induces antidepressant-like effects in rats." Behav Brain Res.

Seese, R. R., L. Y. Chen, C. D. Cox, D. Schulz, A. H. Babayan, W. E. Bunney, F. A. Henn, C. M. Gall and G. Lynch (2013). "Synaptic abnormalities in the infralimbic cortex of a model of congenital depression." J Neurosci 33(33): 13441-13448.

Silvestre, J. S., R. Nadal, M. Pallares and N. Ferre (1997). "Acute effects of ketamine in the holeboard, the elevated-plus maze, and the social interaction test in Wistar rats." Depress Anxiety 5(1): 29-33.

Slaker, M. L., J. H. Harkness and B. A. Sorg (2016). "A standardized and automated method of perineuronal net analysis using Wisteria floribunda agglutinin staining intensity." IBRO Rep 1: 54-60. Slattery, D. A. and J. F. Cryan (2012). "Using the rat forced swim test to assess antidepressant-like activity in rodents." Nat Protoc 7(6): 1009-1014.

Slattery, D. A., I. D. Neumann and J. F. Cryan (2011). "Transient inactivation of the infralimbic cortex induces antidepressant-like effects in the rat." J Psychopharmacol 25(10): 1295-1303.

Trofimiuk, E., P. Wielgat, J. J. Braszko and H. Car (2019). "Stress and Ketamine, Bimodal Influence on Cognitive Functions." Behav Brain Res 360: 354-364.

Wan, Y. Q., J. G. Feng, M. Li, M. Z. Wang, L. Liu, X. Liu, X. X. Duan, C. X. Zhang and X. B. Wang (2018). "Prefrontal cortex miR-29b-3p plays a key role in the antidepressant-like effect of ketamine in rats." Exp Mol Med 50(10): 140.

Warden, M. R., A. Selimbeyoglu, J. J. Mirzabekov, M. Lo, K. R. Thompson, S. Y. Kim, A. Adhikari, K. M. Tye, L. M. Frank and K. Deisseroth (2012). "A prefrontal cortex-brainstem neuronal projection that controls response to behavioural challenge." Nature.

WHO (2017). Depression and Other Common Mental Disorders: Global Health Estimates.

Yuman, N., I. Idaku, Y. Kenkichi, T. Takeshi, O. Kensuke and M. Hiroshi (2008). High-speed video analysis of laboratory rats behaviors in forced swim test. 2008 IEEE International Conference on Automation Science and Engineering.

Zeng, L. L., H. Shen, L. Liu, L. Wang, B. Li, P. Fang, Z. Zhou, Y. Li and D. Hu (2012). "Identifying major depression using whole-brain functional connectivity: a multivariate pattern analysis." Brain 135(Pt 5): 1498-1507.

Zhao, R. R., E. M. Muir, J. N. Alves, H. Rickman, A. Y. Allan, J. C. Kwok, K. C. Roet, J. Verhaagen, B. L. Schneider, J. C. Bensadoun, S. G. Ahmed, R. J. Yanez-Munoz, R. J. Keynes, J. W. Fawcett and J. 
bioRxiv preprint doi: https://doi.org/10.1101/2021.05.30.446326; this version posted May 30, 2021. The copyright holder for this preprint (which

was not certified by peer review) is the author/funder, who has granted bioRxiv a license to display the preprint in perpetuity. It is made available under aCC-BY-NC-ND 4.0 International license.

H. Rogers (2011). "Lentiviral vectors express chondroitinase ABC in cortical projections and promote sprouting of injured corticospinal axons." $\underline{\mathrm{J} \text { Neurosci Methods } 201(1): 228-238 .}$ 